\title{
INVERSE SPECTRAL AND INVERSE NODAL PROBLEMS FOR STURM-LIOUVILLE EQUATIONS WITH POINT $\delta$ AND $\delta^{\prime}$-INTERACTIONS
}

\author{
MANAF DZH. MANAFOV
}

In memory of M. G. Gasymov on his 80th birthday

\begin{abstract}
Inverse spectral and inverse nodal problems are studied for Sturm-Liouville equations with point $\delta$ and $\delta^{\prime}$-interactions. Uniqueness theorems are proved and a constructive procedure for the solutions is provided.
\end{abstract}

\section{Introduction}

We consider the Sturm-Liouville boundary value problem (BVP) $L$ :

$$
\begin{gathered}
l y:=-y^{\prime \prime}+q(x) y=\lambda y, \quad x \in\left(0, \frac{\pi}{4}\right) \cup\left(\frac{\pi}{4}, \frac{\pi}{2}\right) \cup\left(\frac{\pi}{2}, \pi\right), \\
U(y):=y(0)=0, V(y):=y^{\prime}(\pi)=0, \\
I_{1}(y):=\left\{\begin{array}{c}
y\left(\frac{\pi}{4}+0\right)=y\left(\frac{\pi}{4}-0\right)=y\left(\frac{\pi}{4}\right) \\
y^{\prime}\left(\frac{\pi}{4}+0\right)-y^{\prime}\left(\frac{\pi}{4}-0\right)=\alpha y\left(\frac{\pi}{4}\right),
\end{array}\right. \\
I_{2}(y):=\left\{\begin{array}{l}
y\left(\frac{\pi}{2}+0\right)-y\left(\frac{\pi}{2}-0\right)=\beta y^{\prime}\left(\frac{\pi}{2}\right), \\
y^{\prime}\left(\frac{\pi}{2}+0\right)=y^{\prime}\left(\frac{\pi}{2}-0\right)=y^{\prime}\left(\frac{\pi}{2}\right),
\end{array}\right.
\end{gathered}
$$

where $q(x), \alpha, \beta \neq 0$ are real, and $q(x) \in W_{2}^{1}[(0, \pi)], \lambda$ is a spectral parameter.

Notice that, we can understand problem $(1.1),(1.3),(1.4)$ as studying the equation

$$
-y^{\prime \prime}+\left(\alpha \delta\left(x-\frac{\pi}{4}\right)+\beta \delta^{\prime}\left(x-\frac{\pi}{2}\right)+q(x)\right) y=\lambda y, \quad x \in(0, \pi),
$$

where $\delta(x)$ is the Dirac function, and $\delta^{\prime}(x)$ is its derivative function (see [1]).

On the Hilbert space $L_{2}([0, \pi])$ consider the linear differential operator

$$
L: y(x) \rightarrow-y^{\prime \prime}(x)+q(x) y(x)
$$

2000 Mathematics Subject Classification. Primary 34A55,34B24, Secondary 34L05,47E05 .

Key words and phrases. Sturm-Liouville equations, inverse spectral and inverse nodal problems, point $\delta$ and $\delta^{\prime}$-interactions. 
with a dense domain

$$
\begin{aligned}
D(L)= & \left\{y(x) \in W_{2}^{2}\left[(0, \pi) \backslash\left\{\frac{\pi}{4}, \frac{\pi}{2}\right\}\right] \cap W_{2}^{0}[(0, \pi)], y(0)=0,\right. \\
& y\left(\frac{\pi}{4}+0\right)=y\left(\frac{\pi}{4}-0\right), y^{\prime}\left(\frac{\pi}{4}+0\right)-y^{\prime}\left(\frac{\pi}{4}-0\right)=\alpha y\left(\frac{\pi}{4}\right), \\
& y\left(\frac{\pi}{2}+0\right)-y\left(\frac{\pi}{2}-0\right)=\beta y^{\prime}\left(\frac{\pi}{2}\right) \\
& \left.y^{\prime}\left(\frac{\pi}{2}+0\right)=y^{\prime}\left(\frac{\pi}{2}-0\right), y^{\prime}(\pi)=0\right\}
\end{aligned}
$$

We will consider inverse problems of recovering $q(x)$ the given spectral and nodal characteristics. The coefficients $\alpha$ and $\beta$ from (1.3) and (1.4) respectively, are assumed to be known a priori and fixed. We denote the BVP (1.1)-(1.4) by $L=L(q)$.

We study inverse spectral and inverse nodal problems for Sturm-Liouville equations. Inverse spectral problems consist in recovering operators from their spectral characteristics. Such problems play an important role in mathematics and have many applications in natural sciences(see, for example, monographs [3], [12], [15], [17], [23]). Inverse nodal problems consist in constructing operators from the given nodes (zeros) of eigenfunctions (see [11], [14], [16], [19], [24]). BVPs with discontinuities in an interior point also appear in geophysical models for oscillations of the Earth (see [10]). Discontinuous inverse problems (in various formulations) have been considered in [4], [5], [6], [9], [18], [20], [22]. In the present paper we obtain some results on inverse spectral and inverse nodal problems and establish connections between them.

\section{Inverse Spectral Problems}

In this section we study the so-called incomplete inverse problem of recovering the potential $q(x)$ from a part of the spectrum of BVP $L$ provided. We note that for recovering $q(x)$ on the whole interval $(0, \pi)$ it is necessary to specify two spectra of the BVPs with different boundary conditions (see [21]).

Let $y(x)$ and $z(x)$ be continuously differentiable functions on $\left(0, \frac{\pi}{4}\right),\left(\frac{\pi}{4}, \frac{\pi}{2}\right)$ and $\left(\frac{\pi}{2}, \pi\right)$. Denote $\langle y, z\rangle:=y z^{\prime}-y^{\prime} z$. If $y(x)$ and $z(x)$ satisfy the matching conditions (1.3) and (1.4), then

$$
\langle y, z\rangle_{x=\frac{\pi}{4}-0}=\langle y, z\rangle_{x=\frac{\pi}{4}+0},\langle y, z\rangle_{x=\frac{\pi}{2}-0}=\langle y, z\rangle_{x=\frac{\pi}{2}+0},
$$

i.e. the function $\langle y, z\rangle$ is continuous on $(0, \pi)$.

Let $\varphi(x, \lambda)$ be solution of equation (1.1) satisfying the initial conditions

$$
\varphi(0, \lambda)=0, \varphi^{\prime}(0, \lambda)=1
$$

and the matching conditions (1.3),(1.4). Then $U(\varphi)=0$. Denote

$$
\Delta(\lambda)=-V(\varphi)=-\varphi^{\prime}(\pi, \lambda) .
$$

By virtue of (2.1) and the Liouville's formula (see [2],p.83), $\Delta(\lambda)$ does not depend on $x$. The function $\Delta(\lambda)$ is called characteristic function on $L$. 
Theorem 2.1. The BVP L has a countable set of eigenvalues $\left\{\lambda_{n}\right\}_{n \geq 1}$. All eigenvalues are real, simple and $n \rightarrow \infty$

$$
k_{n}:=\sqrt{\lambda_{n}}=n+\frac{1}{2 \pi n}\left(\omega_{0}+(-1)^{n-1} \omega_{1}\right)+o\left(\frac{1}{n}\right),
$$

where

$$
\omega_{0}=\frac{4}{\beta}+\alpha+\int_{0}^{\pi} q(t) d t, \omega_{1}=\alpha+2 \int_{\pi / 2}^{\pi} q(t) d t-\int_{0}^{\pi} q(t) d t .
$$

Proof. Let $\lambda=k^{2}, \tau:=\operatorname{Im} k$. From (2.2), in order to find eigenvalues, we have to construct a solution $\varphi(x, \lambda)$ for all interval $(0, \pi)$. For this reason we construct the following equation

$$
-y^{\prime \prime}+\left(\alpha \delta(x-a)+\beta \delta^{\prime}(x-b)+q(x) y=\lambda y, \quad x \in(0, \pi), \quad(a<b)\right.
$$

which has a solution $\varphi(x, \lambda)$.For $|\lambda| \rightarrow \infty$ uniformly in $x$ one has:

$$
\begin{aligned}
& \varphi(x, \lambda)=\frac{\sin k x}{k}-\frac{\cos k x}{2 k^{2}} \int_{0}^{x} q(t) d t+o\left(\frac{1}{k^{2}} \exp (|\tau| x)\right), x<a, \\
& \varphi^{\prime}(x, \lambda)=\cos k x+\frac{\sin k x}{2 k} \int_{0}^{x} q(t) d t+o\left(\frac{1}{k} \exp (|\tau| x)\right), x<a .
\end{aligned}
$$

Using the following conditions

$$
\left\{\begin{array}{c}
\varphi(a-0, \lambda)=\varphi(a+0, \lambda)=\varphi(a, \lambda) \\
\varphi^{\prime}(a+0, \lambda)-\varphi^{\prime}(a-0, \lambda)=\alpha \varphi(a, \lambda)
\end{array}\right.
$$

we write $\varphi(x, \lambda)$ in $(a, b)$ :

$$
\begin{gathered}
\varphi(x, \lambda)=\frac{\sin k x}{k}-\frac{\cos k x}{2 k^{2}}\left(\int_{0}^{x} q(t) d t+\alpha\right)+\alpha \frac{\cos k(2 a-x)}{2 k^{2}} \\
+o\left(\frac{1}{k^{2}} \exp (|\tau| x)\right), a<x<b, \\
\varphi^{\prime}(x, \lambda)=\cos k x+\frac{\sin k x}{2 k}\left(\int_{0}^{x} q(t) d t+\alpha\right)+\alpha \frac{\sin k(2 a-x)}{2 k} \\
+o\left(\frac{1}{k} \exp (|\tau| x)\right), a<x<b .
\end{gathered}
$$

Using the following conditions

$$
\left\{\begin{array}{l}
\varphi(b+0, \lambda)-\varphi(b-0, \lambda)=\beta \varphi^{\prime}(b, \lambda) \\
\varphi^{\prime}(b+0, \lambda)=\varphi^{\prime}(b-0, \lambda)=\varphi^{\prime}(b, \lambda)
\end{array}\right.
$$

we write $\varphi(x, \lambda)$ in $(b, \pi)$ :

$$
\begin{gathered}
\varphi(x, \lambda)=\frac{\beta}{2}(\cos k x+\cos k(2 b-x))+\frac{\sin k x}{2 k}\left(2+\frac{\beta}{2}\left(\int_{0}^{x} q(t) d t+\alpha\right)\right) \\
+\frac{\sin k(2 b-x)}{2 k}\left(\frac{\beta}{2}\left(\alpha+\int_{0}^{b} q(t) d t-\int_{b}^{x} q(t) d t\right)\right) \\
+\alpha \beta \sin k(2 a-b) \frac{\cos k(x-b)}{2 k}+o\left(\frac{1}{k} \exp (|\tau| x)\right), \quad x>b,
\end{gathered}
$$




$$
\begin{gathered}
\varphi^{\prime}(x, \lambda)=\frac{\beta k}{2}(-\sin k x+\sin k(2 b-x))+\frac{\cos k x}{2}\left(2+\frac{\beta}{2}\left(\int_{0}^{x} q(t) d t+\alpha\right)\right) \\
-\frac{\cos k(2 b-x)}{2}\left(\frac{\beta}{2}\left(\alpha+\int_{0}^{b} q(t) d t-\int_{b}^{x} q(t) d t\right)\right) \\
-\alpha \beta \sin k(2 a-b) \frac{\cos k(x-b)}{2}+o(\exp (|\tau| x)), \quad x>b .
\end{gathered}
$$

When $a=\frac{\pi}{4}$ and $b=\frac{\pi}{2}$, it follows from (2.9) that for $|\lambda| \rightarrow \infty$

$$
\Delta(\lambda)=\frac{\beta}{2}\left(k \sin k \pi-\frac{\omega_{0} \cos k \pi}{2}+\frac{\omega_{1}}{2}\right)+o(\exp (|\tau| \pi)) .
$$

Using (2.10) and Rouche's theorem, by the well-known method (see [3]) for $n \rightarrow$ $\infty$

$$
k_{n}=n+o(1) \text {. }
$$

Analogously, by using Rouche's theorem one can prove that for sufficiently large values of $n$, every circle $\sigma_{n}(\delta)=\{k:|k-n| \leq \delta\}$ contains exactly one zero of $\Delta\left(k^{2}\right)$, namely, $k_{n}=\sqrt{\lambda_{n}}$. Since $\delta>0$ is arbitrary, we must have

$$
k_{n}=n+\varepsilon_{n}, \quad \varepsilon_{n}=o(1), \quad n \rightarrow \infty .
$$

Since $k_{n}$ are zeros of $\Delta\left(k^{2}\right)$, from $(2.10)$ we get

$$
n \sin \varepsilon_{n} \pi-\frac{1}{2}\left(\omega_{0} \cos \varepsilon_{n} \pi+(-1)^{n-1} \omega_{0}\right)+v_{n}=0,
$$

where $v_{n}=\varepsilon_{n} \sin \varepsilon_{n} \pi+o\left(\exp \left(\left|\tau_{n}\right| \pi\right)\right), \tau_{n}=\operatorname{Im} k_{n}$. Hence $\sin \varepsilon_{n} \pi=o\left(\frac{1}{n}\right)$, that is, $\varepsilon_{n}=o\left(\frac{1}{n}\right)$. Using (2.13) we get more precisely

$$
\varepsilon_{n}=\frac{1}{2 \pi n}\left(\omega_{0}+(-1)^{n-1} \omega_{1}\right)+o\left(\frac{1}{n}\right) .
$$

Substituting (2.14) into (2.12), we get (2.2).Since the BVP $L$ is self-adjoint (see [13]), all eigenvalue $\left\{\lambda_{n}\right\}_{n \geq 1}$ are real and simple.

Together with $L$ we consider a BVP $\widetilde{L}=L(\widetilde{q})$ of the same form but with different coefficient $\widetilde{q}$ ( we remind that the coefficients $\alpha$ and $\beta$ from (1.3) and (1.4) respectively, are fixed and known a priori). The following theorem has been proved in [8] for the Sturm- Liouville equation. We show it also holds for (1.1)-(1.4). We assume that $\int_{0}^{\pi / 4} q(x) d x=0$.

Theorem 2.2. If for any $n \in \mathbb{N} \cup\{0\}$

$$
\lambda_{n}=\widetilde{\lambda}_{n},\left\langle y_{n}, \widetilde{y}_{n}\right\rangle_{x=\frac{\pi}{4}-0}=\left\langle y_{n}, \widetilde{y}_{n}\right\rangle_{x=\frac{\pi}{2}-0}=0,
$$

then $q(x)=\widetilde{q}(x)$ almost everywhere (a.e) on $(0, \pi)$, where $\left\langle y_{n}, \widetilde{y}_{n}\right\rangle=$ $y_{n}(x) \widetilde{y}_{n}^{\prime}(x)-y_{n}^{\prime}(x) \widetilde{y}_{n}(x), y_{n}(x)=\varphi\left(x, \lambda_{n}\right)$.

Proof. Since

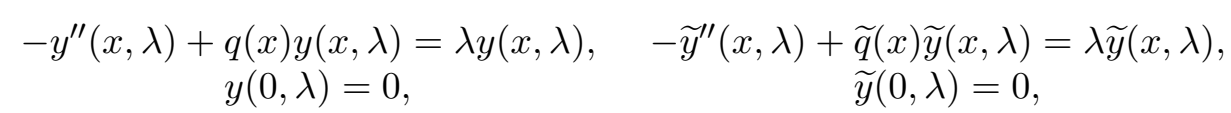


it follows from (2.1) that

$$
\int_{0}^{\frac{\pi}{4}} r(x) y(x, \lambda) \widetilde{y}(x, \lambda) d x=\langle y, \widetilde{y}\rangle_{x=\frac{\pi}{4}-0}
$$

where $r(x)=q(x)-\widetilde{q}(x)$. Since $\left\langle y_{n}, \widetilde{y}_{n}\right\rangle_{x=\frac{\pi}{4}-0}=0$ for $n \in \mathbb{N} \cup\{0\}$, it follows from (2.15) that

$$
\int_{0}^{\frac{\pi}{4}} r(x) y_{n}(x) \widetilde{y}_{n}(x) d x=0, n \in \mathbb{N} \cup\{0\} .
$$

For $x \leq \frac{\pi}{4}$ the following representation holds (see [12], [15]):

$$
y(x, \lambda)=\frac{\sin k x}{k}+\int_{0}^{x} K(x, t) \frac{\sin k t}{k} d t,
$$

where $K(x, t)$ is a continuous function which does not depend on $\lambda$.

Hence

$$
2 k^{2} y(x, \lambda) \widetilde{y}(x, \lambda)=1-\cos 2 k x-\int_{0}^{x} V(x, t) \cos 2 k t d t,
$$

where $V(x, t)$ is a continuous function which does not depend $\lambda$. Substituting (2.17) into (2.16) and taking the relation $\int_{0}^{\pi / 4} r(x) d x=0$ into account, we calculate

$$
\int_{0}^{\frac{\pi}{4}}\left(r(x)+\int_{x}^{\frac{\pi}{4}} V(t, x) r(t) d t\right) \cos 2 k_{n} x d x=0, n \in \mathbb{N} \cup\{0\},
$$

which implies from the completeness of the function cos, that

$$
r(x)+\int_{x}^{\frac{\pi}{4}} V(t, x) r(t) d t=0 \text { a. e. on }\left(0, \frac{\pi}{4}\right) .
$$

But this equation is a homogeneous Volterra integral equation and has only the zero solution, it follows that $r(x)=0$ a. e. on $\left(0, \frac{\pi}{4}\right)$. To prove that $q(x)=\widetilde{q}(x)$ a.e. on $\left[\frac{\pi}{4}, \frac{\pi}{2}\right]$ we will consider the supplementary problem $\widehat{L}$ :

$$
\begin{gathered}
-y^{\prime \prime}(x)+q_{1}(x) y(x)=\lambda y(x), \quad q_{1}(x)=q\left(\frac{\pi}{2}-x\right), \quad 0<x<\frac{\pi}{4}, \\
U(y):=y(0)=0, \\
y\left(\frac{\pi}{4}+0\right)=y\left(\frac{\pi}{4}-0\right), \quad y^{\prime}\left(\frac{\pi}{4}+0\right)-y^{\prime}\left(\frac{\pi}{4}-0\right)=\alpha y\left(\frac{\pi}{4}+0\right) .
\end{gathered}
$$

It follows from (2.1) that

$$
\left\langle y_{n}, \widetilde{y}_{n}\right\rangle_{x=\frac{\pi}{4}+0}=0 .
$$

A direct calculation implies that $\widehat{y}_{n}(x):=y_{n}\left(\frac{\pi}{2}-x\right)$ is the solution to the supplementary problem $\widehat{L}$ and $\widehat{y}_{n}\left(\frac{\pi}{4}-0\right):=y_{n}\left(\frac{\pi}{4}+0\right)$. Thus for the supplementary problem $\widehat{L}$, the assumption conditions in Theorem 2.2 are still satisfied. 
If we repeat the above arguments then this yields $r\left(\frac{\pi}{2}-x\right)=0$ on $0<x<\frac{\pi}{4}$, that is, $q(x)=\widetilde{q}(x)$ a.e. on $\left[\frac{\pi}{4}, \frac{\pi}{2}\right]$.Analogously, to prove that $q(x)=\widetilde{q}(x)$ a.e. on $\left[\frac{\pi}{2}, \pi\right)$ we will consider the supplementary problem $\widehat{\widehat{L}}$ :

$$
\begin{gathered}
-y^{\prime \prime}(x)+q_{2}(x) y(x)=\lambda y(x), \quad q_{2}(x)=q(\pi-x), \quad 0<x<\frac{\pi}{2}, \\
U(y):=y(0)=0, \\
y\left(\frac{\pi}{2}+0\right)-y\left(\frac{\pi}{2}-0\right)=\beta y^{\prime}\left(\frac{\pi}{2}-0\right), \quad y^{\prime}\left(\frac{\pi}{2}+0\right)=y^{\prime}\left(\frac{\pi}{2}-0\right) .
\end{gathered}
$$

If we take the above arguments into consideration, then this implies $r(\pi-x)=0$ on $0<x<\frac{\pi}{2}$, that is, $q(x)=\widetilde{q}(x)$ a.e. on $\left[\frac{\pi}{2}, \pi\right)$. The proof of the theorem is finished.

\section{Inverse Nodal Problems}

In this section, we consider the inverse nodal problems with point $\delta$ and $\delta^{\prime}$ interactions. We obtain uniqueness theorems and a procedure of recovering the potential $q(x)$ on the whole interval $(0, \pi)$ from a dense subset of nodal points. We recall that these results were given for regular Sturm-Liouville problems defined on the interval $(0,1)$ in $[7],[16]$.

The eigenfunctions of the BVP $L$ have the form $y_{n}(x)=\varphi\left(x, \lambda_{n}\right)$. We note that $y_{n}(x)$ are real-valued functions. Substituting (2.3) into (2.4), (2.6) and (2.8) we obtain the following asymptotic formulae for $n \rightarrow \infty$ uniformly in $x$ :

$$
\begin{gathered}
k_{n} y_{n}(x)=\sin n x+\frac{1}{2 \pi n}\left(\begin{array}{rl}
\left.-\pi \int_{0}^{x} q(t) d t+\left(\omega_{0}+(-1)^{n-1} \omega_{1}\right) x\right) \cos n x \\
+o\left(\frac{1}{n}\right), x<\frac{\pi}{4}
\end{array}\right. \\
k_{n} y_{n}(x)=\sin n x+\frac{1}{2 \pi n}\left(-\pi\left(\int_{0}^{x} q(t) d t+\alpha\right)+\left(\omega_{0}+(-1)^{n-1} \omega_{1}\right) x\right) \cos n x \\
+\frac{1}{2 n} \alpha \cos \frac{\pi}{2} n \cos n x+o\left(\frac{1}{n}\right), \frac{\pi}{4}<x<\frac{\pi}{2} \\
y_{n}(x)=\frac{\beta}{2}\left(1+(-1)^{n}\right) \cos n x+\frac{1}{2 \pi n}\left(\pi\left(2+\frac{\beta}{2} \int_{0}^{x} q(t) d t+\alpha\right)\right) \\
+(-1)^{n} \pi\left(\frac{\beta}{2} \int_{0}^{\pi / 2} q(t) d t-\int_{\pi / 2}^{x} q(t) d t\right) \\
-\left(\omega_{0}+(-1)^{n-1} \omega_{1}\right)\left(x+(-1)^{n}(\pi-x)\right) \sin n x+o\left(\frac{1}{n}\right), x>\frac{\pi}{2}
\end{gathered}
$$

For the BVP $L$ an analog of Sturm's oscillation theorem is true. More precisely, the eigenfunction $y_{n}(x)$ has exactly $n-1$ (simple) zeros inside the interval $(0, \pi)$ : $0<x_{n}^{1}<\ldots<x_{n}^{n-1}<\pi$. The set $X_{L}:=\left\{x_{n}^{j}\right\}_{n \geq 2, j=1, n-1}$ is called the set of nodal points of the BVP $L$.

Inverse nodal problems consist in recovering the potential $q(x)$ from the given set $X_{L}$ of nodal points or from a certain its part. Denote $X_{L}^{k}:=\left\{x_{2 m-k}^{j}\right\}_{m \geq 1, j=1,2 m-k-1}, k=0,1$. Clearly, $X_{L}^{0} \cup X_{L}^{1}=X_{L}$. 
Taking (3.1)-(3.3) into account, we obtain the following asymptotic formulae for nodal points as $n \rightarrow \infty$ uniformly in $j$ :

$$
\begin{aligned}
& x_{n}^{j}=\frac{j \pi}{n}+\frac{1}{2 n^{2}}\left(\int_{0}^{j \pi / n} q(t) d t-\frac{\left(\omega_{0}-\omega_{1}\right) j}{n}\right)+o\left(\frac{1}{n^{2}}\right), x_{n}^{j} \in\left(0, \frac{\pi}{4}\right), n=2 m, \\
& x_{n}^{j}=\frac{j \pi}{n}+\frac{1}{2 n^{2}}\left(\int_{0}^{j \pi / n} q(t) d t-\frac{\left(\omega_{0}+\omega_{1}\right) j}{n}\right)+o\left(\frac{1}{n^{2}}\right), x_{n}^{j} \in\left(0, \frac{\pi}{4}\right), n=2 m+1 \text {, } \\
& x_{n}^{j}=\frac{j \pi}{n}+\frac{1}{2 n^{2}}\left(\int_{0}^{j \pi / n} q(t) d t-\frac{\left(\omega_{0}+\omega_{1}\right) j}{n}\right)+o\left(\frac{1}{n^{2}}\right), x_{n}^{j} \in\left(\frac{\pi}{4}, \frac{\pi}{2}\right), n=4 m, \\
& x_{n}^{j}=\frac{j \pi}{n}+\frac{1}{2 n^{2}}\left(\int_{0}^{j \pi / n} q(t) d t-\frac{\left(\omega_{0}-\omega_{1}\right) j}{n}+\alpha\right)+o\left(\frac{1}{n^{2}}\right), x_{n}^{j} \in\left(\frac{\pi}{4}, \frac{\pi}{2}\right), \\
& n=4 m+1, n=4 m+3, \\
& x_{n}^{j}=\frac{j \pi}{n}+\frac{1}{2 n^{2}}\left(\int_{0}^{j \pi / n} q(t) d t-\frac{\left(\omega_{0}+\omega_{1}\right) j}{n}+2 \alpha\right)+o\left(\frac{1}{n^{2}}\right), x_{n}^{j} \in\left(\frac{\pi}{4}, \frac{\pi}{2}\right), \\
& n=4 m+2, \\
& x_{n}^{j}=\left(j-\frac{1}{2}\right) \frac{\pi}{n}+\frac{1}{2 n^{2}}\left(-\int_{0}^{\pi / 2} q(t) d t+\frac{\omega_{0}-\omega_{1}-2}{\beta}-\alpha\right)+o\left(\frac{1}{n^{2}}\right), x_{n}^{j} \in\left(\frac{\pi}{2}, \pi\right) \text {, } \\
& x_{n}^{j}=\frac{1}{2 n^{2}}\left(-\beta \int_{0}^{j \pi / n} q(t) d t+\int_{0}^{\pi / 2} q(t) d t+\frac{2\left(\omega_{0}+\omega_{1}\right) j}{n}-\left(2+\frac{\alpha \beta}{2}+\omega_{0}+\omega_{1}\right)\right) \\
& +o\left(\frac{1}{n^{2}}\right), x_{n}^{j} \in\left(\frac{\pi}{2}, \pi\right), n=2 m+1 .
\end{aligned}
$$

Using these formulas we arrive at the following assertion.

Theorem 3.1. Fix $k=0 \vee 1$ and $x \in[0, \pi]$. Let $\left\{x_{n}^{j_{n}}\right\} \in X_{L}^{k}$ be chosen such that $\lim _{n \rightarrow \infty} x_{n}^{j_{n}}=x$. Then there exists a finite limit

$$
f_{k}(x)=\lim _{n \rightarrow \infty} 2 n\left(x_{n}^{j_{n}} n-j_{n} \pi\right)
$$

and

$$
f_{k}(x)=\left\{\begin{array}{c}
\int_{0}^{x} q(t) d t-\frac{\omega_{0}+(-1)^{k+1} \omega_{1}}{\pi} x, x \leq \frac{\pi}{4} \\
\int_{0}^{x} q(t) d t-\frac{\omega_{0}+(-1)^{k+1} \omega_{1}}{\pi} x-\alpha \cos \frac{\pi}{2} k, \frac{\pi}{4} \leq x \leq \frac{\pi}{2}, \\
\frac{1}{2}\left(\int_{0}^{x} q(t) d t+(-1)^{k+1} \int_{0}^{x} q(t) d t\right)-(-1)^{k} \int_{0}^{\pi / 2} q(t) d t \\
-\frac{\omega_{0}+(-1)^{k+1} \omega_{1}}{\beta \pi} x+\frac{\omega_{0}+(-1)^{k+1}}{\beta}-\alpha, x \geq \frac{\pi}{2}
\end{array}\right.
$$


Let us now formulate a uniqueness theorem and provide a constructive procedure for the solution of the inverse nodal problem.

Theorem 3.2. Fix $k=0 \vee 1$. Let $X \subset X_{L}^{k}$ be a subset of nodal points which is dense on $(0, \pi)$. Let $X=\widetilde{X}$. Then $q(x)=\widetilde{q}(x)$ a.e. on $(0, \pi)$. Thus the specification of $X$ uniquely determines the potential $q(x)$ on $(0, \pi)$. The function $q(x)$ can be constructed via the formulae

$$
q(x)=f_{k}^{\prime}(x)-\frac{1}{\pi}\left(f_{k}(\pi)+f_{k}\left(\frac{\pi}{2}\right)-f_{k}(0)-\alpha\right),
$$

where $f_{k}(x)$ is calculated by (3.5).

Proof. Formula (3.6) follows from (3.5). Note that if $X=\tilde{X}$, then (3.4) yields $f_{k}(x) \equiv \widetilde{f}_{k}(x), x \in(0, \pi)$. By virtue of $(3.6)$, we get $q(x)=\widetilde{q}(x)$ a.e. on $(0, \pi)$.

\section{References}

[1] S. Albeverio, F. Gesztesy, R. Hoegh-Krohn, H. Holden, with an appendix by P. Exner, Solvable Models in Quantum Mechanics (second edition), AMS Chelsea Publ., 2005.

[2] E. A. Coddington, N. Levinson, Theory of ordinary differential equations, McGrawHill, New York, USA, 1955.

[3] G. Freiling, V. A. Yurko, Inverse Sturm-Liouville Problems and Their Applications, NOVA Science Publishers, New York, 2001.

[4] G. Freiling, V. A. Yurko, Inverse spectral problems for singular non-selfadjoint differential operators with discontinuities in an interior point, Inverse Problems $\mathbf{1 8}$ (2002), 757-773.

[5] I. M. Guseinov, L. I. Mammadova, Reconstruction of the diffusion equation with singular coefficients for two spectra, Dokl. Acad. Nauk, 457 (1) (2014), 13-16; Eng. transl: Dokl. Math., 90 (1) (2014), 401-404.

[6] O. H. Hald, Discontinuous inverse eigenvalue problems, Comm. Pure Appl. Math. 37 (1984), 539-577.

[7] O. H. Hald, J. R. McLaughlin, Solutions of inverse nodal problems, Inverse problems 5 (1989), 307-347.

[8] M. Horvath, Inverse spectral problems and closed exponential systems, Ann. of Math. 162 (2005), 885-918.

[9] R. O. Hryniv, Ya. V. Mykytyuk, Inverse spectral problems for Sturm-Liouville operators with singular potentials, Inverse Problems 19 (2003), 665-684.

[10] F. R. Lapwood, T. Usami, Free Oscillations of the Earth, Cambridge Univ. Press, Cambridge, 1981.

[11] C. K. Law, C. F. Yang, Reconstucting the potential function and its derivatives using nodal data, Inverse Problems 14(2) (1998), 299-312.

[12] B. M. Levitan, Inverse Sturm-Liouville Problems, Nauka, Mockow, 1984; Eng. Transl.: VNU Sci. Press, Utrecht, 1987.

[13] M. Dzh. Manafov, Description of the domain of an ordinary differential operator with generalized potentials, Differ. Uravneniya 32:5 (1996), 706-707; Eng. transl.: Differential Equations 32(5) (1996), 716-718.

[14] M. Dzh. Manafov, Inverse spectral and inverse nodal problems for Sturm-Liouville equations with point $\delta^{\prime}$-Interaction, Trans. of NAS of Azerb., Series of PhysicalTech. and Math. Sci., Issue Math., 37 (4) (2017), 111-119.

[15] V. A. Marchenko, Sturm-Liouville Operators and Their Applications , Naukova Dumka, Kiev, 1977; English Transl., Birkhöuser, 1986. 
[16] J. R. McLaughlin, Inverse spectral theory using nodal points as data-a uniqueness result, J. Differential Equations 73 (1988), 354-362.

[17] J. Pöschel, E. Trubowitz, Inverse Spectral Theory, Academic Press, New York, 1987.

[18] A. M. Savchuk, A mapping method in inverse Sturm-Liouville problems with singular potentials, Trudy Mat. Ins. Steklova 261 (2008), 243-248; Eng. transl.: Proceedings of the Steklov Ins. of Math. 261 (2008), 237-242.

[19] C. L. Shen, C. T. Chieh, An inverse nodal problem for vectorial Strum-Liouville equation, Inverse Problems 16 (2000), 349-356.

[20] D. G. Shepelsky, The inverse problem of reconstuction of the medium's conductivity in a class of discontinuous and increasing functions, in: Spectral Operator Theory and Related Topics. Advances in Soviet Math. 19, Amer. Math. Soc., Providence, RI, 1994, 209-232.

[21] V. A. Yurko, Integral transforms connected with discontinuous boundary value problems, Integral Transforms Spec. Funct. 10(2) (2000), 141-164.

[22] V. A. Yurko, On boundary value problems with jump conditions inside the interval. Differ. Uravneniya 36(8) (2000), 1139-1140; Eng. Transl.: Differential Equations 36(8) (2000), 1266-1269.

[23] V. A. Yurko, Method of Spectral Mappings in the Inverse Problem Theory, Inverse III-posed Probl. Ser., VSP, Utrecht, 2002.

[24] V. A. Yurko, On inverse nodal and spectral problems for boundary value problems with discontinuity conditions inside the interval, Izv. Saratov Univ. Mat. Mekh. Inform. 8(1) (2008), 31-35 (in Russian).

Manaf Dzh. Manafov

Adiyaman University, Faculty of Arts and Sciences, Department of Mathematics, Adryaman, 02040, Turkey

Institute of Mathematics and Mechanics, Azerbaijan National Academy of Sciences, Baku, AZ1141, Azerbaijan

E-mail address: mmanafov@adiyaman.edu.tr

Received: June 28, 2019; Accepted: August 28, 2019 